\title{
Topological groups of bounded homomorphisms on a topological group
}

\author{
Ljubiša D.R. Kočinac; Omid Zabeti \\ University of Niš, Faculty of Sciences and Mathematics, \\ 18000 Niš, Serbia \\ Ikocinac@gmail.com \\ Faculty of Mathematics, University of Sistan and Baluchestan, \\ P.O. Box 98135-674, Zahedan, Iran \\ o.zabeti@gmail.com
}

\begin{abstract}
We consider a few types of bounded homomorphisms on a topological group. These classes of bounded homomorphisms are, in a sense, weaker than the class of continuous homomorphisms. We show that with appropriate topologies each class of these homomorphisms on a complete topological group forms a complete topological group.
\end{abstract}

2010 Mathematics Subject Classification: 22A05, 54H11.

Keywords: Bounded homomorphism; continuous homomorphism; topological group; completeness.

\section{Introduction}

When one deals with the concept of a bounded set in a topological vector space, there are some tools which are absolutely handy: for example, the scalar multiplication and absorbing zero neighborhoods. But when we want to consider the notion of a bounded set in a topological group, the situation is completely different. There are neither scalar field nor absorbing neighborhoods. One may define a bounded subset in a topological group by taking group multiplication instead of scalar multiplication in a topological vector space; but this approach does not match our intuition for a bounded subset, since, for example, the multiplicative group $S^{1}$ is not bounded in this definition.

Following [1, a subset $B$ of a topological group $G$ is called bounded if for each neighborhood $U$ of the identity element $e_{G}$ of $G$ there is a positive integer $n$ such that $B \subset U^{n}$.

\footnotetext{
*Corresponding author
} 
We give here a result concerning this kind of boundedness in the products of topological groups.

Theorem 1.1 Let $\left\{G_{\alpha}: \alpha \in \Lambda\right\}$ be a set of abelian topological groups and $G=\prod_{\alpha \in \Lambda} G_{\alpha}$ with the product topology. Then $B \subseteq G$ is bounded if and only if there are finitely many sets $B_{\alpha_{1}}, \ldots, B_{\alpha_{k}}$ such that for each $i \leq k, B_{\alpha_{i}}$ is a bounded set in $G_{\alpha_{i}}$ and $B \subset \prod_{i \leq k} B_{\alpha_{i}} \times \prod_{\alpha \in \Lambda \backslash\left\{\alpha_{1}, \ldots, \alpha_{k}\right\}} G_{\alpha}$.

Proof. Suppose $B \subseteq G$ is bounded. Put

$$
B_{\alpha_{i}}=\left\{x \in G_{\alpha_{i}}: \exists \mathbf{y}=\left(y_{\alpha}\right) \in B \text { and } x \text { is } \alpha_{i} \text {-th coordinate of } \mathbf{y}\right\} .
$$

Each $B_{\alpha_{i}}, i \leq k$, is bounded. For, if $U_{i}, i \leq k$, is a neighborhood of the identity in $G_{\alpha_{i}}$ put

$$
U=U_{\alpha_{1}} \times \ldots \times U_{\alpha_{k}} \times \prod_{\alpha \neq \alpha_{i}} G_{\alpha} .
$$

$U$ is a neighborhood of the identity in $G$. Therefore, there is a positive integer $n$ with $B \subseteq U^{n}$, so that $B_{\alpha_{i}} \subseteq U_{i}^{n}$. It is easy to see that $B \subseteq B_{\alpha_{1}} \times \ldots B_{\alpha_{k}} \times$ $\prod_{\alpha \in \Lambda \backslash\left\{\alpha_{1}, \ldots, \alpha_{k}\right\}} G_{\alpha}$.

Suppose now that for a set $B \subseteq G$, there exist sets $B_{\alpha_{1}}, \ldots, B_{\alpha_{k}}$ such that $B_{\alpha_{i}}$ is a bounded set in $G_{\alpha_{i}}, i \leq k$, and $B \subset \prod_{i \leq k} B_{\alpha_{i}} \times \prod_{\alpha \in \Lambda \backslash\left\{\alpha_{1}, \ldots, \alpha_{k}\right\}} G_{\alpha}$. Let $U$ be an arbitrary neighborhood of the identity element of $G$; without loss of generality, we may assume that $U$ is of the form

$$
U=U_{1} \times \ldots \times U_{k} \times \prod_{\alpha \in \Lambda \backslash\left\{\alpha_{1}, \ldots, \alpha_{k}\right\}} G_{\alpha}
$$

where $U_{i}$ is a neighborhood of the identity element in $G_{\alpha_{i}}, i \leq k$. For each $i \leq k$ there is a positive integer $n_{i}$ such that $B_{\alpha_{i}} \subset U_{i}^{n_{i}}$. Put $n=\max \left\{n_{1}, \ldots, n_{k}\right\}$. Evidently $B \subset U^{n}$, i.e. $B$ is bounded in $G$.

Bounded homomorphisms and their algebraic and topological structures on any topological algebraic structure are of interest for their own rights and also for their applications in other areas of mathematics. For examples, bounded operators on a topological vector space with suitable topologies form topological algebras; also, there is a spectral theory for these classes of bounded operators with some useful applications (see [3, 6, 7] for ample information). Therefore, it will be of interest to consider different types of bounded homomorphisms on a topological group and to investigate which topological and algebraic properties of the underlying topological group can be transferred to the mentioned classes of homomorphisms. In this note, we examine a few notions of bounded homomorphisms on a topological group. These classes of bounded homomorphisms contain continuous homomorphisms. We equip each class of such bounded homomorphisms by an appropriate topology. It turns out that they constitute complete topological groups provided that the underlying topological group is assumed to be complete and its singletons are bounded. 
For terminology and notations used in this paper, we refer the reader to [4, as well as for an abstract and comprehensive taste of topological groups and related notions. All topological groups in this note are assumed to be abelian. It is well known that a topological group has a local base at the identity element consisting of symmetric neighborhoods. Throughout the paper we consider only such bases. The identity element of a group $G$ will be denoted by $e_{G}$.

\section{Results}

Definition 2.1 Let $G$ and $H$ be two topological groups. A homomorphism $T: G \rightarrow H$ is said to be

(1) nb-bounded if there exists a neighborhood $U$ of $e_{G}$ such that $T(U)$ is bounded in $H$;

(2) bb-bounded if for every bounded set $B \subset G, T(B)$ is bounded in $H$.

The set of all nb-bounded (bb-bounded) homomorphisms from a topological group $G$ to a topological group $H$ is denoted by $\operatorname{Hom}_{\mathrm{nb}}(G, H)\left(\operatorname{Hom}_{\mathrm{bb}}(G, H)\right)$. We write $\operatorname{Hom}(G)$ instead of $\operatorname{Hom}(G, G)$.

Remark 2.2 For topological groups $G$ and $H$ the following holds:

$$
\operatorname{Hom}_{\mathrm{nb}}(G, H) \subset \operatorname{Hom}_{\mathrm{bb}}(G, H) .
$$

Let $T: G \rightarrow H$ be an nb-bounded homomorphism. Then it is bb-bounded. For, suppose $B \subset G$ is a bounded set. Since $T$ is nb-bounded there is a neighborhood $U$ of $e_{G}$ such that $T(U)$ is bounded in $H$. Boundedness of $B$ implies $B \subset U^{n}$ for some natural number $n$. We prove that $T(B)$ is bounded in $H$. Let $V$ be a neighborhood of $e_{H}$. Boundedness of $T(U)$ implies that there is $m \in \mathbb{N}$ such that $T(U) \subset V^{m}$. Then

$$
T(B) \subset T\left(U^{n}\right)=(T(U))^{n} \subset V^{m n},
$$

i.e. $T(B)$ is bounded in $H$.

Note that the converse is not true as the following example shows.

Example 2.3 Let $G=\mathbb{C}-\{0\}$ be the group of non-zero complex numbers, and $H=G^{\mathbb{N}}$, the group of all sequences of elements of $G$, with the pointwise multiplication and the product topology. Consider the identity homomorphism $1_{H}$ on $H$. It is easy to see that $1_{H}$ is bb-bounded, but it is not nb-bounded since $H$ is not locally bounded.

Remark 2.4 One may verify that each continuous homomorphism is bb-bounded. Let $G$ and $H$ be topological groups, $T: G \rightarrow H$ be a continuous homomorphism, and $B$ a bounded subset of $G$. Suppose a neighborhood $V$ of $e_{H}$ is given. There 
exists a neighborhood $U$ of $e_{G}$ such that $T(U) \subset V$. Also, since $B$ is bounded in $G$, there is an $n \in \mathbb{N}$ with $B \subset U^{n}$. Thus,

$$
T(B) \subset T\left(U^{n}\right)=(T(U))^{n} \subset V^{n} .
$$

Nevertheless, unlike in the case of bounded operators on topological vector spaces, there is no more relation between continuous homomorphisms on a topological group and bounded ones, which is explained in the example below.

Example 2.5 Let $G$ be $S^{1}$, the multiplicative group of all complex numbers of modulus 1, with the trivial (anti-discrete) topology, and $H$ be $S^{1}$ with the topology inherited from $\mathbb{C}$. Consider the identity homomorphism $i$ from $G$ into $H$. Clearly, $i$ is not continuous. But it is nb-bounded, as well as bb-bounded.

Remark 2.6 Note that if $G$ is a topological vector space over $\mathbb{R}$ or $\mathbb{C}$, we have two notions for a bounded set; one of them is related to the group structure of $G$ and another considers the scalar multiplication. It is easy to see that these aspects of bounded sets have the same meaning. by

Let $G$ be a group. For homomorphisms $T$ and $S$ on $G$, define $T S$ and $T^{-1}$

$$
T S(x):=T(x) S(x) \text { and } T^{-1}(x):=(T(x))^{-1}, x \in G .
$$

It is easy to see that with these operations, the class of all homomorphisms on a group $G$ forms a group.

Now, assume $G$ is a topological group. The class of all nb-bounded homomorphisms on $G$ equipped with the topology of uniform convergence on some neighborhood of $e_{G}$ is denoted by $\operatorname{Hom}_{\mathrm{nb}}(G)$. Observe that a net $\left(S_{\alpha}\right)$ of nbbounded homomorphisms converges uniformly on a neighborhood $U$ of $e_{G}$ to a homomorphism $S$ if for each neighborhood $V$ of $e_{G}$ there exists an $\alpha_{0}$ such that for each $\alpha \geq \alpha_{0},\left(S_{\alpha} S^{-1}\right)(U) \subset V$.

The class of all bb-bounded homomorphisms on $G$ endowed with the topology of uniform convergence on bounded sets is denoted by $\operatorname{Hom}_{\mathrm{bb}}(G)$. Note that a net $\left(S_{\alpha}\right)$ of bb-bounded homomorphisms uniformly converges to a homomorphism $S$ on a bounded set $B \subset G$ if for each neighborhood $V$ of $e_{G}$ there is an $\alpha_{0}$ with $\left(S_{\alpha} S^{-1}\right)(B) \subset V$ for each $\alpha \geq \alpha_{0}$.

The class of all continuous homomorphisms on $G$ equipped with the topology of c-convergence is denoted by $\operatorname{Hom}_{\mathrm{c}}(G)$. A net $\left(S_{\alpha}\right)$ of continuous homomorphisms c-converges to a homomorphism $S$ if for each neighborhood $W$ of $e_{G}$, there is a neighborhood $U$ of $e_{G}$ such that for every neighborhood $V$ of $e_{G}$ there exists an $\alpha_{0}$ with $\left(S_{\alpha} S^{-1}\right)(U) \subset V W$ for each $\alpha \geq \alpha_{0}$.

Note that $\operatorname{Hom}_{\mathrm{c}}(G)$ and $\operatorname{Hom}_{\mathrm{bb}}(G)$ form subgroups of the group of all homomorphisms on $G$. On the other hand, $\operatorname{Hom}_{\mathrm{nb}}(G)$ is not a group, in general; the identity homomorphism on $G$ in Example 2.3 does not belong to $\operatorname{Hom}_{\mathrm{nb}}(G)$.

Theorem 2.7 The operations of multiplication and inversion are continuous in $\mathrm{Hom}_{\mathrm{nb}}(G)$ (with respect to the topology of uniform convergence on some neighborhood of $\left.e_{G}\right)$. 
Proof. Suppose $\left(T_{\alpha}\right)$ and $\left(S_{\alpha}\right)$ are two nets of nb-bounded homomorphisms which are convergent uniformly on some neighborhood $U$ of $e_{G}$ to the nbbounded homomorphisms $T$ and $S$, respectively. Let $W$ be an arbitrary neighborhood of $e_{G}$. There is a neighborhood $V$ of $e_{G}$ with $V V \subset W$. There exist some $\alpha_{1}$ and $\alpha_{2}$ such that $\left(T_{\alpha} T^{-1}\right)(U) \subset V$ for each $\alpha \geq \alpha_{1}$ and $\left(S_{\alpha} S^{-1}\right)(U) \subset$ $V$ for each $\alpha \geq \alpha_{2}$. Choose $\alpha_{0}$ with $\alpha_{0} \geq \alpha_{1}$ and $\alpha_{0} \geq \alpha_{2}$. If $\alpha \geq \alpha_{0}$, then we have

$$
\left(T_{\alpha} S_{\alpha}\right)(T S)^{-1}(U) \subset\left(T_{\alpha} T^{-1}\right)(U)\left(S_{\alpha} S^{-1}\right)(U) \subset V V \subset W .
$$

Also, because of continuity of the inversion we have

$$
\left(T_{\alpha} T^{-1}\right)^{-1}(U)=\left(T_{\alpha} T^{-1}(U)\right)^{-1} \subset W,
$$

for sufficiently large $\alpha$. This completes the proof.

Theorem 2.8 The operations of multiplication and inversion are continuous in $\mathrm{Hom}_{\mathrm{bb}}(G)$ with respect to the topology of uniform convergence on bounded sets.

Proof. Suppose $\left(T_{\alpha}\right)$ and $\left(S_{\alpha}\right)$ are two nets of bb-bounded homomorphisms which are convergent uniformly on bounded sets to the bb-bounded homomorphisms $T$ and $S$, respectively. Fix a bounded set $B \subset G$. Let $W$ be an arbitrary neighborhood of $e_{G}$. There exists a neighborhood $V$ of $e_{G}$ with $V V \subset W$. There are some $\alpha_{1}$ and $\alpha_{2}$ such that $\left(T_{\alpha} T^{-1}\right)(B) \subset V$ for each $\alpha \geq \alpha_{1}$ and $\left(S_{\alpha} S^{-1}\right)(B) \subset V$ for each $\alpha \geq \alpha_{2}$. Choose $\alpha_{0}$ with $\alpha_{0} \geq \alpha_{1}$ and $\alpha_{0} \geq \alpha_{2}$. If $\alpha \geq \alpha_{0}$, then we have

$$
\left(T_{\alpha} S_{\alpha}\right)(T S)^{-1}(B) \subset\left(T_{\alpha} T^{-1}\right)(B)\left(S_{\alpha} S^{-1}\right)(B) \subset V V \subset W .
$$

Since the inversion in $G$ is continuous, for sufficiently large $\alpha$ we have

$$
\left(T_{\alpha} T^{-1}\right)^{-1}(B)=\left(T_{\alpha} T^{-1}(B)\right)^{-1} \subset W
$$

which completes the proof.

Theorem 2.9 The operations of multiplication and inversion are continuous in $\operatorname{Hom}_{\mathrm{c}}(G)$, i.e. $\operatorname{Hom}_{\mathrm{c}}(G)$ is a topological group.

Proof. Suppose $\left(T_{\alpha}\right)$ and $\left(S_{\alpha}\right)$ are two nets of continuous homomorphisms $c$-converging to the homomorphisms $T$ and $S$, respectively. Let $W$ and $V$ be arbitrary neighborhoods of $e_{G}$. There exist neighborhoods $W_{1}$ and $V_{1}$ of $e_{G}$ such that $W_{1} W_{1} \subset W$ and $V_{1} V_{1} \subset V$. There are a neighborhood $U$ of $e_{G}$ and some indices $\alpha_{1}$ and $\alpha_{2}$ with $\left(T_{\alpha} T^{-1}\right)(U) \subset V_{1} W_{1}$ for each $\alpha \geq \alpha_{1}$ and $\left(S_{\alpha} S^{-1}\right)(U) \subset V_{2} W_{2}$ for each $\alpha \geq \alpha_{2}$. Choose $\alpha_{0}$ such that $\alpha_{0} \geq \alpha_{1}$ and $\alpha_{0} \geq \alpha_{2}$. If $\alpha \geq \alpha_{0}$, then we have

$$
\left(T_{\alpha} S_{\alpha}\right)(T S)^{-1}(U) \subset\left(T_{\alpha} T^{-1}\right)(U)\left(S_{\alpha} S^{-1}\right)(U) \subset V_{1} W_{1} V_{2} W_{2} \subset V W .
$$

Now, continuity of the inversion implies

$$
\left(T_{\alpha} T^{-1}\right)^{-1}(U)=\left(T_{\alpha} T^{-1}(U)\right)^{-1} \subset V W,
$$


for sufficiently large $\alpha$. This completes the proof.

In this part, we investigate whether or not each class of bounded homomorphisms in the assumed topology is uniformly complete.

Lemma 2.10 Suppose $\left(S_{\alpha}\right)$ is a net of continuous homomorphisms which converges to the homomorphism $S$ in the c-convergence topology. Then, $S$ is also continuous.

Proof. Let an arbitrary neighborhood $W$ of $e_{G}$ be given. Choose a neighborhood $V$ of $e_{G}$ such that $V^{3} \subset W$. There are a neighborhood $U$ of $e_{G}$ and an $\alpha_{0}$ with $\left(S_{\alpha} S^{-1}\right)(U) \subset V^{2}$ for each $\alpha \geq \alpha_{0}$. Fix an $\alpha \geq \alpha_{0}$. There exists a neighborhood $U_{1} \subset U$ such that $S_{\alpha}\left(U_{1}\right) \subset V$. From here, together with $\left(S_{\alpha} S^{-1}\right)\left(U_{1}\right) \subset V^{2}$, it follows

$$
S\left(U_{1}\right) \subset S_{\alpha}\left(U_{1}\right) V^{2} \subset V^{3} \subset W,
$$

as desired.

Lemma 2.11 If $\left(S_{\alpha}\right)$ is a net of bb-bounded homomorphisms which converges to the homomorphism $S$ in the topology of uniform convergence on bounded sets. Then $S$ is bb-bounded.

Proof. Let $W$ be an arbitrary neighborhood of $e_{G}$. Fix a bounded set $B \subset G$. There is an $\alpha_{0}$ such that $\left(S_{\alpha} S^{-1}\right)(B) \subset W$ for each $\alpha \geq \alpha_{0}$. Fix an $\alpha \geq \alpha_{0}$. There exists a natural $n$ with $S_{\alpha}(B) \subset W^{n}$, so that

$$
S(B) \subset\left(S_{\alpha}(B)\right) W \subset W^{n} W=W^{n+1},
$$

as we wanted.

Remark 2.12 The class $\operatorname{Hom}_{\mathrm{nb}}(G)$ can contain a Cauchy sequence whose limit is not an $n b$-bounded homomorphism; in other words, $\mathrm{B}_{\mathrm{nb}}(G)$ is not uniformly complete in the assumed topology. Let $G$ be as in Example 2.3 and $\left(S_{n}\right)$ be a sequence of homomorphisms on $G$ which are defined as follows:

$$
S_{n}\left(\left(x_{n}\right)\right)=\left(x_{1}, \ldots, x_{n}, 1, \ldots\right) .
$$

Each $S_{n}$ is nb-bounded. For, if $U_{n}$ is the neighborhood of $e_{G}$ defined by

$$
U_{n}=\left\{\left(x_{n}\right),\left|x_{i}-1\right| \leq \frac{1}{2}, i=1,2, \ldots, n\right\},
$$

then, as it is easy to see, $S_{n}\left(U_{n}\right)$ is bounded in $G$. On the other hand, it is not difficult to show that $\left(S_{n}\right)$ is uniformly convergent to the identity homomorphism $1_{G}$ on $G$. But we have seen in Example 2.3 that $1_{G}$ is not nb-bounded.

Now, we are going to find conditions under which each class of considered bounded homomorphisms is topologically complete. In the case of bounded 
operators on topological vector spaces, absorbing neighborhoods and local convexity are two fruitful tools for discovering conditions (see [3]). In the topological group version, it turns out that boundedness of every singleton is a handy tool. By [4, Theorem 7.4], when $G$ is a connected topological group, then it is absorbed by positive powers of any neighborhood of $e_{G}$, so that every singleton is bounded. Note that connectedness is not a necessary condition; for example, the additive group $\mathbb{Q}$ of rational numbers is totally disconnected, but every its singleton is bounded. There are examples of abelian topological groups whose singletons are not bounded. For example, let $G$ be an abelian topological group and consider the topological group $H=G \times \mathbb{Z}_{2}$. Then $G \times\{0\}$ is a zero neighborhood which is not absorbing, so that singletons are not bounded.

In what follows we assume that every singleton in the underlying topological group $G$ is bounded.

Theorem 2.13 If $G$ is a complete group, then $\operatorname{Hom}_{\mathrm{c}}(G)$ is complete.

Proof. Suppose $\left(T_{\alpha}\right)$ is a Cauchy net in $\operatorname{Hom}_{\mathrm{c}}(G)$ and $W$ is an arbitrary neighborhood of $e_{G}$. Choose a neighborhood $V$ of $e_{G}$ with $V V \subset W$. Find a neighborhood $U$ of $e_{G}$ and an index $\alpha_{0}$ such that $\left(T_{\alpha} T_{\beta}{ }^{-1}(U)\right) \subset V V$ for each $\alpha \geq \alpha_{0}$ and for each $\beta \geq \alpha_{0}$. Suppose $x \in G$.

First, assume that $x \in U$. Then $T_{\alpha} T_{\beta}{ }^{-1}(x) \in W$. Thus, $\left(\left(T_{\alpha}(x)\right)\right.$ is a Cauchy net in $G$.

For an arbitrary $x \in G$, there is a positive integer $n$ such that $x \in U^{n}$. Since the product of Cauchy nets in a topological group is again Cauchy, it follows that $\left(T_{\alpha}(x)\right)$ is a Cauchy net in $G$, so that it converges. Put $T(x):=\lim T_{\alpha}(x)$ for each $x \in G$. Since this convergence holds in $\operatorname{Hom}_{\mathrm{c}}(G)$, by Lemma 2.10, $T$ is also continuous, and this completes the proof.

Theorem 2.14 If $G$ is a complete group, then $\operatorname{Hom}_{\mathrm{bb}}(G)$ is also complete.

Proof. Suppose $\left(T_{\alpha}\right)$ is a Cauchy net in $\operatorname{Hom}_{\mathrm{bb}}(G)$ and $W$ is an arbitrary neighborhood of $e_{G}$. One can find a neighborhood $V$ of $e_{G}$ such that $V V \subset W$. Since every singleton $x \in G$ is bounded, there is an $\alpha_{0}$ such that $T_{\alpha} T_{\beta}{ }^{-1}(x) \in V$ for each $\alpha \geq \alpha_{0}$ and each $\beta \geq \alpha_{0}$. One concludes that $\left(\left(T_{\alpha}(x)\right)\right.$ is a Cauchy net in $G$. Therefore, it converges. Put $T(x):=\lim T_{\alpha}(x)$, for each $x \in G$. Now fix a bounded set $B \subset G$. For sufficiently large $\alpha$ and $\beta$, we have $T_{\alpha} T_{\beta}{ }^{-1}(B) \subset V$, so that for each $x \in B$,

$$
T_{\alpha} T_{\beta}^{-1}(x) \in V
$$

For sufficiently large $\beta$ we have $T_{\beta}(x) T^{-1}(x) \in V$, and therefore

$$
T_{\alpha} T^{-1}(x)=T_{\alpha} T_{\beta}^{-1}(x) T_{\beta}(x) T^{-1}(x) \in V V \subset W .
$$

Since this convergence holds in $\operatorname{Hom}_{\mathrm{bb}}(G)$, by Lemma 2.11, $T$ is also bb-bounded, as required.

Remark 2.15 Note that when $G$ is a complete group, then $\operatorname{Hom}_{\mathrm{nb}}(G)$ might fail to be a complete topological group. Consider Example 2.3 and Remark 2.12. 


\section{Conclusion}

We considered here two kinds of bounded homomorphisms in topological groups. It would be interesting to consider other sorts of bounded homomorphisms as well. Recall that a subset $B$ of a topological groups $G$ is $\aleph_{0}$-bounded if for each neighborhood $U$ of $e_{G}$ there is a countable set $C$ such that $B \subset C U ; B \subset G$ is Menger-bounded (or shortly M-bounded) if for each sequence $\left(U_{n}: n \in \mathbb{N}\right.$ ) of neighborhoods of $e_{G}$ there is a sequence $\left(F_{n}: n \in \mathbb{N}\right)$ of finite subsets of $G$ such that $B \subset \bigcup_{n \in \mathbb{N}} F_{n} U_{n}$. If in the last definition $F_{n}$ s are singletons, then $B$ is said to be Rothberger-bounded (shortly R-bounded (see [2, 5, for more details about these classes of sets). A homomorphism $T$ between topological groups $G$ and $H$ is said to be:

(i) wb-bounded if for each $\aleph_{0}$-bounded subset $B$ of $G, T(B)$ is bounded in $H$;

(ii) mb-bounded if for each M-bounded subset $B$ of $G, T(B)$ is bounded in $H$;

(iii) rb-bounded if for each R-bounded subset $B$ of $G, T(B)$ is bounded in $H$.

\section{Acknowledgement}

We are grateful to the referees for useful remarks and suggestions.

\section{References}

[1] K. Haghnejad Azar, Bounded topological groups, Preprint.

[2] L. Babinkostova, Lj.D.R. Kočinac, M. Scheepers, Combinatorics of open covers (XI): Menger- and Rothberger-bounded groups, Topology Appl. 154:7 (2007) 1269-1280.

[3] S. Hejazian, M. Mirzavaziri, O. Zabeti, Bounded operators on topological vector spaces and their spectral radii, Filomat 26:6 (2012) 1283-1290

[4] E. Hewitt, K.A. Ross, Abstract Harmonic Analysis, Springer, Berlin, 1963.

[5] Lj.D.R. Kočinac, Star selection principles: A survey, Khayyam J. Math. 1:1 (2015) 82-106.

[6] V.G. Troitsky, Spectral radii of bounded linear operators on topological vector spaces, J. PanAmerican Math. 11:3 (2001) 1-35.

[7] O. Zabeti, Topological algebras of bounded operators on topological vector spaces, J. Adv. Res. Pure Math. 3:1 (2011) 22-26. 